\title{
THERMONUCLEAR BURN CRITERIA
}

\author{
Guido Van Oost $^{1}$ and Roger Jaspers ${ }^{2}$
}

\author{
1. Department Applied Physics \\ Ghent University \\ St. Pietersnieuwstraat 41, B-9000 Gent, Belgium \\ Guido.Vanoost@ugent.be \\ 2. Science and Technology of Nuclear Fusion, \\ Faculty of Applied Physics, \\ Eindhoven University of Technology, \\ P.O. Box 513, 5600 MB Eindhoven, The Netherlands
}

\section{INTRODUCTION}

After more than 50 years of fusion research the time has arrived when fusion processes in experimental plasmas are increasingly getting important. In JET the genuine fuel (deuterium-tritium) of a fusion reactor was used for the first time in late 1991, in TFTR the same happened in 1993, and in JET an extended period of experiments of this kind was performed in 1997. Therefore, it is getting more and more rewarding to deal with the problems related to the ignition and burning of plasmas.

Nuclear fusion played and still plays an important role in the Universe. About 1 million years after the bigbang large amounts of ${ }^{4} \mathrm{He}$ were created by the fusion of protons on a global scale, and later on heavier elements were and are created in the huge fusion reactors provided by the interior of the stars. On earth, the concepts envisaged for a fusion reactor are thermonuclear fusion by magnetic plasma confinement in tokamaks or stellarators, laser- or beam-induced inertial fusion, and muon catalyzed cold fusion. In this lecture we shall concentrate on magnetic confinement, in particular on the D-T fusion reaction ${ }^{2} \mathrm{D}+{ }^{3} \mathrm{~T} \rightarrow{ }^{4} \mathrm{He}+\mathrm{n}$, which has a mass defect $\Delta m=m_{\mathrm{D}}^{+}+m_{\mathrm{T}}^{+}-\left(m_{4_{\mathrm{He}}}^{+}+m_{n}\right)=$ $3.1 \cdot 10^{-29} \mathrm{~kg}$, i.e. about 4 per thousand of the reactant's mass, that according to Einstein's equation $E=m c^{2}$ corresponds to an energy $E=17.6 \mathrm{MeV}$ released as kinetic energy in the reaction products. Starting from the 5 nucleons in the D\&T nuclei, this means $3.5 \mathrm{MeV}$ per nucleon or about 4 times the $0.85 \mathrm{MeV}$ which is released per nucleon during the fission of U235. The distribution of fusion energy among the reaction products is determined by the momentum conservation law. Since the momentum of the reaction products is much larger than that of the reaction partners before the reaction, in a D-T reaction we essentially have $m_{\mathrm{n}} v_{\mathrm{n}}=-m_{\mathrm{He}} v_{\mathrm{He}}$.
From this, with $E=m v^{2} / 2$ we immediately obtain

$$
E_{\mathrm{n}} / E_{\mathrm{He}}=m_{\mathrm{He}} / m_{\mathrm{n}}=4
$$

Usually this process is described by the chemical notation

$$
{ }^{2} \mathrm{D}+{ }^{3} \mathrm{~T} \rightarrow{ }^{3} \mathrm{He}(3.5 \mathrm{MeV})+{ }^{1} \mathrm{n}(14.1 \mathrm{MeV})
$$

Since the binding energy B of the nucleons (neutrons and protons) must be expended for their separation from the nucleus, it is released in the reverse process, fusion. And since each nucleus possesses negative binding energy, its mass is always smaller than the sum of the masses of all neutrons and protons (total number A) of which it consists. In Fig. 1 we see how B/A depends on $\mathrm{A}$. In the range $A \leq 60$ the average binding energy per nucleon can be increased (brought to larger negative values) by the fusion of smaller nuclei into larger ones; in the range $A \geq 60$ the same effect is achieved by the fission of larger nuclei into smaller fragments.

While the first fusion reactions had already been observed in 1919 by the physicist Ernest Rutherford, nuclear fission was only discovered in 1938 by the two chemists Otto Hahn and Fritz Strassmann. Nevertheless it was only four years until the physicist Enrico Fermi obtained the first controlled chain reactions in an experimental fission reactor. On the other hand we shall have to wait far into this century until the first fusion reactor will hopefully go into operation. This is an indication of how much more difficult it is to obtain controlled fusion reactions with an net energy gain. The obstacles in nuclear fusion are well illustrated by the following estimate: The energy needed to overcome the Coulomb-wall of mutual repulsion for two hydrogen nuclei is about $0.4 \mathrm{MeV}$, and the temperature of a plasma needed 


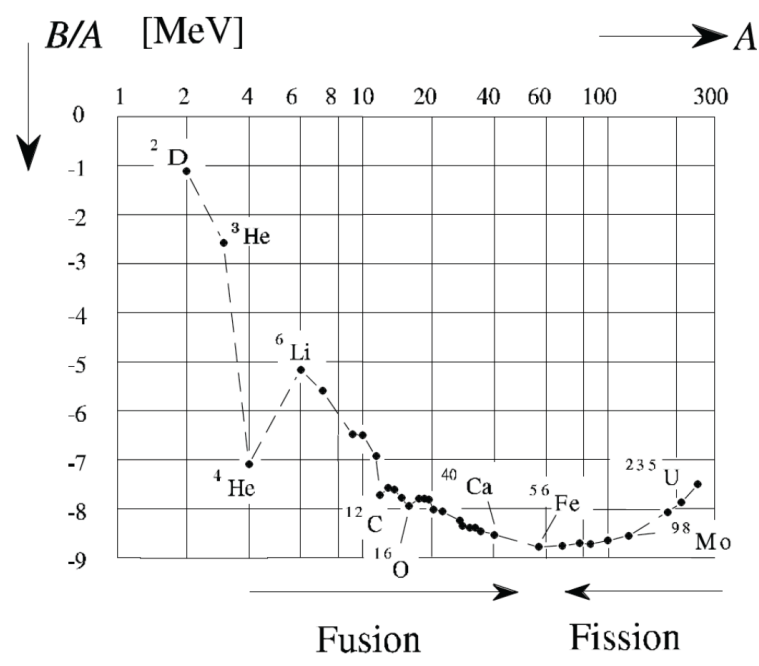

Figure 1: Binding energy per nucleon, B/A, as a function of $\mathrm{A}$

for the particles to achieve this in a classical process with the help of their thermal energies is $T \approx 3 \cdot 10^{9}$ K. Fortunately the tunnelling effect makes considerably lower temperatures possible. In a D-T reaction the quantum probability for penetrating the Coulomb wall is given by the Gamow factor

$$
w \approx \exp \left(-34.4 \sqrt{\mathrm{keV} / E_{\mathrm{kin}}}\right)
$$

For $E_{\text {kin }}=10 \mathrm{keV}$ we obtain a tunnelling probability of $w \approx 1.9 \cdot 10^{-5}$, indicating that markedly lower temperatures than the classically required 3 billion Kelvin can lead to fusion. All this is included in the fusion reaction cross-section below (Section II).

According to the curve of binding energies the direct fusion of the ${ }^{4} \mathrm{He}$-nucleus out of its four nucleons would be even more energetic than the D-T reaction because a total binding energy of $28 \mathrm{MeV}$ would be released in this process, i.e. $7 \mathrm{MeV}$ per nucleon. However, a reaction of this kind would require the simultaneous collision of four nuclei, a process that is so highly improbable at normal densities in magnetic fusion that it practically does not occur. Indeed, as we have seen before, the fusion of two reaction partners is already a rather improbable process, so only two-particle collisions can be envisaged for fusion reactions in a reactor.

Altogether more than 80 different fusion reactions are currently known. Since singly charged nuclei have the lowest Coulomb repulsion, fusion reactions between hydrogen isotopes require the lowest plasma temperatures. The D-T reaction (2) is accompanied by a number of side-reactions, the most important of which are D-D and T-T reactions. However, we will neglect these side reactions because of their small fusion crosssections.
In the $\mathrm{D}-\mathrm{T}$ reaction the main portion of the energy is released to the neutron. Although the fast fusion neutrons created this way lead to secondary radioactivity in some materials surrounding the plasma (first wall, supports, etc.), in magnetic confinement schemes this must, at least at present, be considered an advantage. Since they don't carry electric charge the neutrons are not held back by the confining magnetic field, and they can also easily penetrate the confinement vessel. Outside of this their energy can be extracted by a moderator.

\section{CROSS-SECTIONS, REACTION RATES AND POWER DENSITY OF FUSION REACTIONS}

A fusion reaction which releases a lot of energy but occurs very rarely is of little use. Thus the reaction frequency is a crucial issue. Let us consider a beam of D-nuclei with density $n_{\mathrm{D}}$, moving at constant relative velocity $\mathrm{v}$ through T-nuclei. The number $\mathrm{d} n_{\mathrm{D}}$ of beam particles that is lost due to interaction processes such as scattering collisions or fusion reactions when the beam advances by a distance $d s$ is proportional to ds, to the density $n_{\mathrm{T}}$ of target particles and to that of the beam particles, $n_{\mathrm{D}}$ :

$$
\mathrm{d} n_{\mathrm{D}}=\sigma n_{\mathrm{D}} n_{\mathrm{T}} \mathrm{d} s \Longrightarrow R:=\dot{n}_{\mathrm{D}}=\dot{n}_{\mathrm{T}}=n_{\mathrm{D}} n_{\mathrm{T}}\langle\sigma(v) v\rangle,
$$

where \langle\rangle denotes the averaging over particles of all possible velocities. $R$ is the reaction rate (or the collision frequency in the case of scattering collisions; for both processes independently a corresponding equation applies). $\sigma$ is the $\mathrm{D}$-T fusion cross-section, $v$ the relative velocity between the reacting particles. The evaluation of the average rate coefficient $\langle\sigma(v) v\rangle$ requires some thermodynamics, involves the Gamow factor, and yields the results shown in Fig. 2 for some typical fusion reactions. It is seen that it assumes by far the largest values in the $\mathrm{D}$ - $\mathrm{T}$ reaction, and this even at much lower temperatures than in the other fusion reactions.

It is only a small fraction of highly energetic particles that are reacting and being lost through fusion (see eq. 3). This tail is repopulated by scattering collisions that cause the plasma to approach a Maxwellian distribution closely. This collisional process for the replacement of highly energetic particles lost by fusion is an essential characteristic of thermonuclear fusion. Thus while scattering collisions have the unpleasant side effect of causing diffusion and particle losses from the reaction vessel on the one hand, on the other hand they have the important task of replenishing highly energetic particles lost by fusion. In a fusion reactor each fusion collision will be accompanied by a sufficiently high number of scattering collisions. Closer investigation shows that at the temperature of a fusion reactor $(\approx 10 \mathrm{keV})$ 


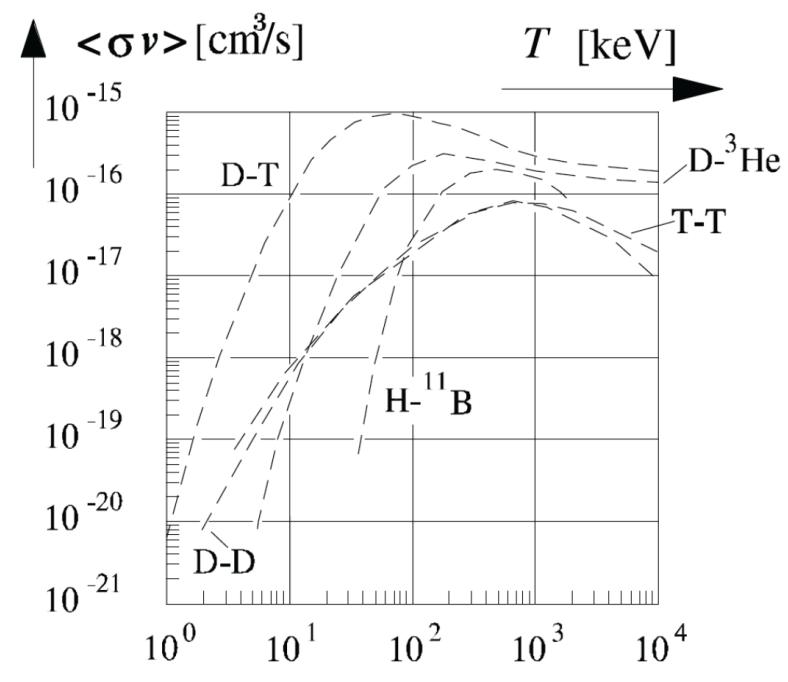

Figure 2: Temperature dependence of the rate coefficient for some typical fusion reactions

on average for each fusion collision there are about 8000 scattering collisions (Note: this is the reason that beamtarget fusion concepts will not produce net energy, and a thermonuclear approach is needed).

The quantity which characterizes the efficiency of a fusion reaction is the power density $P_{\text {fus }}$, the energy released per second in a unit volume:

$$
P_{\text {fus }}=R E_{\text {fus }}=n_{\mathrm{D}} n_{\mathrm{T}}\langle\sigma v\rangle E_{\text {fus }}, \quad E_{\text {fus }}=17.6 \mathrm{MeV}
$$

Both ions and electrons exert a pressure $p_{\mathrm{I}}$ and $p_{\mathrm{e}}$, respectively, adding to a total pressure $p$,

$$
\begin{gathered}
p_{\mathrm{I}}=n_{\mathrm{I}} k T_{\mathrm{I}}=\left(n_{\mathrm{D}}+n_{\mathrm{T}}\right) k T_{\mathrm{I}}, \\
p_{\mathrm{e}}=n_{\mathrm{e}} k T_{\mathrm{e}}, \quad p=p_{\mathrm{I}}+p_{\mathrm{e}}
\end{gathered}
$$

Due to stability reasons, there is an upper limit $\beta_{\max }$ to the ratio between average plasma pressure and magnetic pressure

$$
\beta=\langle p\rangle /\left\langle B / 2 \mu_{0}\right\rangle
$$

From this and (6)-(7) it follows that there is an upper limit to the fusion particle density that for $n_{\mathrm{D}}=$ $n_{\mathrm{T}} n_{\mathrm{I}} / 2$ and $n_{\mathrm{e}}=n_{\mathrm{D}}+n_{\mathrm{T}}$ (quasi-neutrality) is given by $\left\langle\hat{n}_{\mathrm{I}}\right\rangle \approx\left\langle B^{2} \beta_{\max } /\left(8 \mu_{0} k T\right)\right\rangle$. The maximum fusion power density associated with this is

$$
\hat{P}_{\text {fus }}=\left\langle\frac{B^{4} \beta^{2}}{64 \mu_{0}^{2} k^{2}} \frac{\langle\sigma v\rangle}{T^{2}} E_{\text {fus }}\right\rangle
$$

Figure 3 shows $\hat{P}_{\text {fus }}$ for a given $\beta_{\max }$ as a function of the temperature for several fusion reactions. Comparison with Fig. 2 reveals that the highest power output is

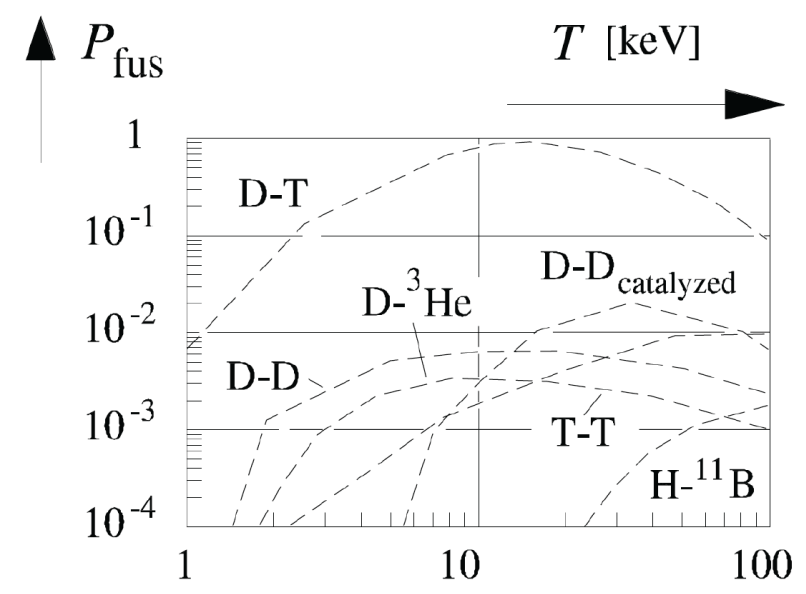

Figure 3: Maximum fusion power density $P_{\text {fus }}[\mathrm{W} / \mathrm{cm} 3]$ vs. $T$.

obtained at a much lower temperature than the maximum value of $\langle\sigma v\rangle$. This is due to the factor $1 / T^{2}$ in $\hat{P}_{\text {fus }}$ that, with increasing temperature, causes $\hat{P}_{\text {fus }}$ to decrease before $\langle\sigma v\rangle$ has reached its maximum value. Equation (9) also demonstrates the importance of high magnetic fields.

\section{BALANCE EQUATIONS}

A. Particle balance

A general particle balance equation has the form

$$
\partial n_{k} / \partial t+\operatorname{div}\left(n_{k} v_{k}\right)=Q_{k}
$$

$n_{k} v_{k}$ is the current of particles consisting of a diffusive and a convective part, and $Q_{k}$ is a local source term. The equation accounts for (a) particle supply, (b) particle gains and losses through the burning of the fuel, and (c) for losses by diffusion and convection. Averaging eq. (10) over the whole plasma volume V (with surface $\mathrm{S}_{\mathrm{V}}$ ) yields

$$
\mathrm{d} \bar{n}_{k} / \mathrm{d} t+\int_{S_{V}} n_{k} v_{k} \cdot \mathrm{d} S / V=\bar{Q}_{k}
$$

where $\bar{n}_{k}=\int n_{k} \mathrm{~d} V / V$ is the average particle density and $\bar{Q}_{k}=\int Q_{k} \mathrm{~d} V / V$ the average source term. $\int n_{K}$. $\mathrm{d} V / V=\bar{n}_{k} \cdot V$ is the total number of particles $\mathrm{N}_{\mathrm{K}}$ (i.e. the particle content).

The source term is composed of the fuel losses described by (4) and a term $\bar{s}_{k}$ accounting for the fuel supply: $\bar{Q}_{i}=\overline{-n_{i} n_{j}\langle\sigma v\rangle}+\bar{s}_{i}$. After multiplication with $\mathrm{V}$ the total loss of particles per second from the plasma, $\mathrm{d} N_{k}^{\text {loss }} / \mathrm{d} t=\int n_{k} v_{k} \cdot \mathrm{d} S$, yields an average particle loss 
rate per volume. This leads to the definition of a particle loss time $\tau_{k}$ through

$$
\tau_{k}=\frac{\bar{n}_{k} V}{\int n_{k} v_{k} \cdot \mathrm{d} S}
$$

Its precise meaning can be seen from the reformulation

$$
\tau_{k} \int_{V} n_{k} v_{k} \cdot \mathrm{d} S=\tau_{k} \mathrm{~d} N_{k}^{\text {loss }} / \mathrm{d} t=\bar{n}_{k} V=N_{k}
$$

Under stationary conditions obtained when all particle losses are compensated for by supply, $\tau_{k}$ is the time elapsed until just as many particles are lost from the plasma through diffusion and convection as it momentarily contains. (We assume that effects of particle recycling[12] are included in $\tau_{k}$ ). Assuming approximately equal diffusion loss times, $\tau_{i}=\tau_{j}=\tau_{p}$, and using the approximations

$$
\overline{n_{i} n_{j}\langle\sigma v\rangle} \approx \bar{n}_{i} \bar{n}_{j} \overline{\langle\sigma v\rangle}, \quad \overline{\langle\sigma v\rangle(T)} \approx\langle\sigma v\rangle(\bar{T})
$$

from (11) and (12) we obtain the burn equations

$$
\mathrm{d} n_{i} / \mathrm{d} t=-n_{i} / \tau_{p}-n_{i} n_{j}\langle\sigma v\rangle+s_{i}
$$

where the volume-averaging bars have been omitted for further convenience. These "zero-dimensional" equations can be improved by taking into account profile effects: For profiles of a given (not self-consistently determined) shape each term is modified by a shape factor (see e.g. Ref. 2).

Equations (14) must be supplemented by the quasineutrality condition

$$
\sum n_{k} Z_{k}=n_{\mathrm{e}}
$$

in which $Z_{k}$ is the charge number of ion species $k$.

\section{B. Energy balance}

With the simplifying assumption $T_{\mathrm{e}}=T_{\mathrm{D}}=T_{\mathrm{T}}=$ $T$ (this implies that all ions created by fusion are thermalized) the general energy balance equation has the form

$$
\frac{\partial}{\partial t} \frac{3}{2}\left(n_{\mathrm{e}}+\sum_{\lambda} n_{\lambda}\right) k T+\operatorname{div} J=p_{\mathrm{OH}}+p_{\mathrm{ext}}+p_{\alpha}+p_{\mathrm{rad}}
$$

where $J$ is the total heat flow current due to heat convection and heat conduction, is the ohmic heating power, $p_{\mathrm{OH}}$ the additional external heating power, $p_{\alpha}$ the alpha particle heating power; the work $v_{\mathrm{e}} \cdot \nabla p_{\mathrm{e}}+$ $\sum_{\lambda} v_{\lambda} \nabla p_{\lambda}$ performed by the pressures has been neglected in comparison with the much larger heat source terms.

1. Ohmic heating: At fusion temperatures $P_{\mathrm{OH}}=$ $\eta j^{2}$ can usually be neglected in comparison with $p_{\alpha}$ since $\eta \propto 1 / T^{3 / 2}$ (this would not be possible in tokamaks with extremely strong magnetic fields because in these much stronger currents would be allowed).

2. External heating: It is useful to express the external heating power as a fraction of the fusion power through

$$
p_{\text {ext }}=p_{\text {fus }} / Q=5 p_{\alpha} / Q
$$

$Q$ is called the power enhancement factor (see also IV). It is the ratio of the thermonuclear power produced to the heating power supplied and is a measure of the success in approaching reactor condition.

3. Alpha particle heating: In our calculations we shall assume that the energy released to the alpha particles through fusion processes is fully delivered to the plasma through collisions. The heating power thus obtained is approximately given by (5) with $E_{\text {fus }}$ replaced by $E_{\alpha}$, i.e.

$$
P_{\alpha}=n_{\mathrm{D}} n_{\mathrm{T}}\langle\sigma v\rangle E_{\alpha}=n_{\mathrm{D}} n_{\mathrm{T}}\langle\sigma v\rangle E_{\mathrm{fus}} / 5
$$

This is only an approximation for the following reasons:

(a) Some alpha particles may already diffuse out, before they have delivered their surplus energy to the plasma.

(b) The expression for $P_{\alpha}$ is a function of the position and time of alpha particle creation; however, the real position and time of energy deposition are somewhat apart or later respectively. Due to $P_{\alpha}=E_{\text {fus }} / 5$ the temperature dependence of $P_{\alpha}$ is the same as that of $P_{\text {fus }}$ shown in Fig. 3.

4. Radiation losses: There are radiation losses through bremsstrahlung, synchrotron radiation, and through line and recombination radiation. At the temperatures of a D-T reactor, $10-20 \mathrm{keV}$, synchrotron radiation can be neglected in comparison with bremsstrahlung. For bremsstrahlung, which originates mainly from the acceleration of electrons in the field of ions, we employ the formula

$$
P_{B}=\frac{e^{6}}{24 \pi e_{0}^{3} c^{3} m_{e} h} n_{e}^{2} Z^{2} \sqrt{\frac{8 k T_{e}}{\pi m_{e}}} g_{\mathrm{ff}}\left(\frac{Z^{2}}{T_{e}}\right)
$$

in which $g_{\mathrm{ff}}$ is a slowly varying function of its argument called Gaunt-factor (accounting for quantum effects). In the D-T reaction there are separate contributions of this kind from $\mathrm{D}$ and $\mathrm{T}$ with charge number $Z=1$ and from ${ }^{4} \mathrm{He}$ with $Z=2$ (helium 
is fully ionised under reactor conditions).

In a pure $\mathrm{D}-\mathrm{T}$ plasma line and recombination radiation do not play an essential role except for the much cooler plasma boundary region, because all ions are fully ionized and the central plasma is too hot for recombinations. The situation is different if the plasma is polluted by nuclei of higher charge number. We shall only rather crudely take into account such radiation, employing for it again eq. (19) with some effective charge number for the impurities.

5. Transport losses: Integrating the heat flow $J$ through diffusion and convection of energy over the plasma boundary yields the total energy losses by transport.

By analogy with (12) we introduce an energy confinement time $\tau_{\mathrm{E}}$ through

$$
\tau_{\mathrm{E}}=\frac{\int_{V} \frac{3}{2}\left(n_{e}+\sum_{\lambda} n_{\lambda}\right) k T \cdot \mathrm{d} V}{\int_{S_{\mathrm{V}} J \cdot \mathrm{d} S}}
$$

Frequently, especially by experimentalists, a different energy confinement time $\tau_{\mathrm{E}}^{*}$ (called global confinement time) is used that is defined through

$$
\tau_{\mathrm{E}}^{*}=\frac{\int_{V} \frac{3}{2}\left(n_{e}+\sum_{\lambda} n_{\lambda}\right) k T \cdot \mathrm{d} V}{P_{\mathrm{rad}}+\int_{S_{\mathrm{V}}} J \cdot \mathrm{d} S}
$$

It is the time in which the plasma, due to all losses including radiation, loses the same amount of energy as it presently contains and is easier to measure than $\tau_{\mathrm{E}}$.

6. Averaged energy balance equation: Integrating eq.(16) over the whole plasma volume, dividing by $V$ and using (17), (20) plus the same approximations as in (13), with omission of the bar for averages we obtain

$$
\begin{array}{r}
\frac{\mathrm{d}}{\mathrm{d} t} e_{\mathrm{tot}}=P_{\alpha}\left(1+\frac{5}{Q}\right)-\frac{e_{\mathrm{tot}}}{\tau_{\mathrm{E}}}-P_{\mathrm{rad}}= \\
P_{\alpha}\left(1+\frac{5}{Q}\right)-\frac{e_{\mathrm{tot}}}{\tau_{\mathrm{E}}^{*}}
\end{array}
$$

Where $e_{\text {tot }}=\left(n_{e}+\sum_{\lambda} n_{\lambda}\right) k T$ is the total energy density and the expressions for $P_{\alpha}$ and $P_{\text {rad }}$ must be evaluated at the average temperature and density.

\section{Basic equations for the $\mathrm{D}-\mathrm{T}$ reaction}

We now make a further approximation in neglecting all side reactions (D-D, T-T etc.) due to their small fusion cross-sections. With this we obtain from (14) the particle balance equations

$$
\frac{\mathrm{d} n_{\mathrm{D}}}{\mathrm{d} t}=-\frac{n_{\mathrm{D}}}{\tau_{p}}-n_{\mathrm{D}} n_{\mathrm{T}}\langle\sigma v\rangle_{\mathrm{DT}}+s_{\mathrm{D}}
$$

$$
\begin{gathered}
\frac{\mathrm{d} n_{\mathrm{T}}}{\mathrm{d} t}=-\frac{n_{\mathrm{T}}}{\tau_{p}}-n_{\mathrm{D}} n_{\mathrm{T}}\langle\sigma v\rangle_{\mathrm{DT}}+s_{\mathrm{T}} \\
\frac{\mathrm{d} n_{\alpha}}{\mathrm{d} t}=-\frac{n_{\alpha}}{\tau_{\alpha}}-n_{\mathrm{D}} n_{\mathrm{T}}\langle\sigma v\rangle_{\mathrm{DT}}
\end{gathered}
$$

and from (22) and (18) the energy balance equation

$$
\begin{aligned}
\frac{\mathrm{d}}{\mathrm{d} t} & {\left[\frac{3}{2}\left(n_{e}+n_{I}+n_{\alpha}+n_{Z}\right) k T\right]=} \\
& -\frac{3}{2}\left(n_{e}+n_{I}+n_{\alpha}+n_{Z}\right) k T / \tau_{\mathrm{E}} \\
+ & n_{\mathrm{D}} n_{\mathrm{T}}\langle\sigma v\rangle_{\mathrm{DT}} E_{\alpha}(1+5 / Q)-P_{B}
\end{aligned}
$$

where $n_{I}$ is the total number of ions and $n_{Z}$ is the density of impurity ions considered as a single species with effective charge number $Z$. We shall consider $n_{Z}$ as a given parameter. In contrast to our previous intentions, we have introduced a separate particle confinement time $\tau_{\alpha} \neq \tau_{p}$ for the alpha particles, the purpose being that this will facilitate the transition to a limiting case to be considered (see IV.A). In addition, we have the quasi-neutrality condition

$$
n_{I}+2 n_{\alpha}+Z n_{Z}=n_{e}=n_{\text {tot }} / 2
$$

For $P$ we have to take into account the radiation caused by hydrogen isotopes $(Z=1)$, alpha particles $(Z=2)$ and impurities (charge number $Z$ ), from (19) obtaining the formula

$$
P_{B}=n_{e}^{2}\left[c_{I} R_{I}(T)+c_{\alpha} R_{\alpha}(T)+c_{Z} R_{Z}(T)\right]
$$

in which we employed the concentrations

$$
c_{I}=\frac{n_{I}}{n_{e}}, c_{\alpha}=\frac{n_{\alpha}}{n_{e}}, c_{Z}=\frac{n_{Z}}{n_{e}}
$$

and where

$$
\begin{aligned}
& R_{I}=C_{B} \sqrt{T} g_{\mathrm{ff}}(1 / T), R_{\alpha}=4 C_{B} \sqrt{T} g_{\mathrm{ff}}(4 / T), \\
& R_{Z}=Z^{2} C_{B} \sqrt{T} g_{\mathrm{ff}}\left(Z^{2} / T\right)
\end{aligned}
$$

with

$$
C_{B}=\frac{e^{6} \sqrt{8 k}}{24 \pi \epsilon_{0}^{3} c^{3} m_{e} h \sqrt{\pi m_{e}}}
$$

\section{EQUILIBRIA: BREAK-EVEN AND IGNITION}

We now want to determine equilibria, i.e. we are looking for stationary solutions $\mathrm{d} / \mathrm{d} t=0$. When an equilibrium is achieved with $P_{\text {ext }}=P_{\text {fus }}$ or $Q=1$ resp. this is called break-even. Ignition (notice the analogy with the burning of fossil fuels) is achieved when all external heat sources can be turned off, $P_{\text {ext }}=0$ or $Q=\infty$. The confinement conditions are then such that the plasma temperature can be maintained against the 
energy losses solely by $\alpha$-particle heating. From (23)(24) for stationary conditions we get

$$
\begin{gathered}
s_{D}=\frac{n_{\mathrm{D}}}{\tau_{p}}+n_{\mathrm{D}} n_{\mathrm{T}}\langle\sigma v\rangle_{\mathrm{DT}}+s_{\mathrm{D}}, \\
s_{T}=\frac{n_{\mathrm{T}}}{\tau_{p}}+n_{\mathrm{D}} n_{\mathrm{T}}\langle\sigma v\rangle_{\mathrm{DT}}+s_{\mathrm{D}}
\end{gathered}
$$

the magnitude of the particle sources is fixed by the requirement of stationarity. The maximum fusion power is obtained for $n_{D}=n_{T}=n_{I} / 2$ (see II), i.e. the particle sources must satisfy

$$
s_{D}=s_{T}=\frac{n_{I}}{2 \tau_{p}}+\frac{n_{I}^{2}}{4}\langle\sigma v\rangle
$$

With this equations (23) and (24) are satisfied and must no longer be considered concerning equilibrium.

The remaining equations to be solved are (25), (26) and (15) viz

$$
c_{I}+2 c_{\alpha}+Z c_{Z}=1
$$

The latter one is satisfied when we eliminate $c_{I}$ by using $c_{I}=1-2 c_{\alpha}-Z c_{Z}$. Inserting this we are left with only two equations (for particles and energy respectively),

$$
\begin{gathered}
\frac{c_{\alpha} n_{e}}{\tau_{\alpha}}=\frac{1}{4}\left(1-2 c_{\alpha}-Z c_{Z}\right)^{2} n_{e}^{2}\langle\sigma v\rangle, \\
\frac{3}{2}\left[2-c_{\alpha}-(Z-1) c_{Z}\right] n_{e} k T / \tau_{\mathrm{E}}= \\
\frac{1}{4}\left(1-2 c_{\alpha}-Z c_{Z}\right)^{2} n_{e}^{2}\langle\sigma v\rangle E_{\alpha}(1+5 / Q)-P_{B}
\end{gathered}
$$

A. Ideal ignition condition, minimum burn temperature, and ideal break-even

In a first quantitative approach we shall neglect the presence of impurities as well as that of the helium ash, i.e. we set $c_{\alpha}=0, c_{Z}=0$. This way we not only get a widely used result for the ignition condition but also one which is very easily comprehensible. Of course this can only be a rough approximation because the accumulation of helium ash can, in principle, not be avoided. $c_{\alpha}=0$ is compatible with the equilibrium equations if in (35) we set $\tau_{\alpha}$ and don't consider this equation any longer. (This is the reason why we introduced a separate confinement time $\tau_{\alpha}$.) For the bremsstrahlung we have $P_{B}=n_{e}^{2} R_{I}(T)$ and the only equation left is the energy equation (36) which becomes

$$
3 n_{e} k T / \tau_{\mathrm{E}}=\frac{1}{4} n_{e}^{2}\langle\sigma v\rangle E_{\alpha}(1+5 / Q)-n_{e}^{2} R_{I}(T)
$$

Dividing it by $n_{e}^{2}$ and then solving it with respect to $n_{e} \tau_{\mathrm{E}}$ we finally obtain the ideal ignition criterion

$$
n_{e} \tau_{\mathrm{E}}=\frac{3 k T}{\frac{1}{4}\langle\sigma v\rangle E_{\alpha}(1+5 / Q)-R_{I}(T)}
$$

The product $n_{e} \tau_{\mathrm{E}}$ is a measure of the quality of the plasma confinement, and the value required according to this formula in order to get an ignited equilibrium or break-even depends only on the temperature. This temperature dependence is shown in Fig. 4. The minimum temperature required for ignition $(Q=\infty)$ is obtained by equating the denominator of our result for $n_{e} \tau_{\mathrm{E}}$ to zero (becoming infinite). It is given by the smaller temperature obtained as a solution from

$$
R_{I}(T)=\frac{1}{4}\langle\sigma v\rangle E_{\alpha}
$$

and is typically about $6 \mathrm{keV}$.

We shall now transform the ideal ignition curve into a diagram employing our second energy confinement time $\tau_{\mathrm{E}}^{*}$ defined in (21). Applying this definition,

$$
P_{\text {rad }}+e_{\text {tot }} / \tau_{\mathrm{E}}=e_{\text {tot }} / \tau_{\mathrm{E}}^{*}
$$

to the present situation yields

$$
\begin{gathered}
n_{e}^{2} R_{I}+3 n_{e} k T / \tau_{\mathrm{E}}=3 n_{e} k T / \tau_{\mathrm{E}}^{*} \\
\tau_{\mathrm{E}}=\frac{3 k T \tau_{\mathrm{E}}^{*}}{3 k T-n_{e} \tau_{\mathrm{E}}^{*} R_{I}}
\end{gathered}
$$

Since $\tau_{\mathrm{E}}$ must be nonnegative, from this we get the condition

$$
n_{e} \tau_{\mathrm{E}}^{*} \leq 3 k T / R_{I}
$$

The limit $n_{e} \tau_{\mathrm{E}}^{*} \leq 3 k T / R_{I}$ is called radiation limit because $\tau_{\mathrm{E}}=\infty$ for it, and all losses are due to radiation. With (42) and multiplication by $T$ the condition (38) transforms into

$$
n_{e} \tau_{\mathrm{E}}^{*} T=\frac{12 k T^{2}}{\langle\sigma v\rangle E_{\alpha}(1+5 / Q)}
$$

The so-called fusion product $n_{e} \tau_{\mathrm{E}}^{*} T$ employed in this formula is widely used for characterizing the performance of a fusion device because it combines the two quantities $n_{e} \tau_{\mathrm{E}}^{*}$ (also a measure for the quality of confinement) and $T$, which both have to be large for ignition, into a single quantity. Its temperature dependence is shown in Fig. 4 together with the radiation limit. According to (43) only states below the radiation limit are physically meaningful. The two intersection points between the radiation limit and the ignition curve describe radiative equilibria. The temperature at the left point is the minimum temperature for which ignition is possible (about $4.4 \mathrm{keV}$ ). Please note that this is only true for plasmas which are transparent for the radiation losses considered; the sun burns at lower temperatures. In the temperature range of a fusion reactor a good approximation for $\langle\sigma v\rangle$ is provided by [13]

$$
\langle\sigma v\rangle=1.1 \times 10^{-24} T^{2} \mathrm{~ms}^{-1}, \quad T \text { in } \mathrm{eV}
$$




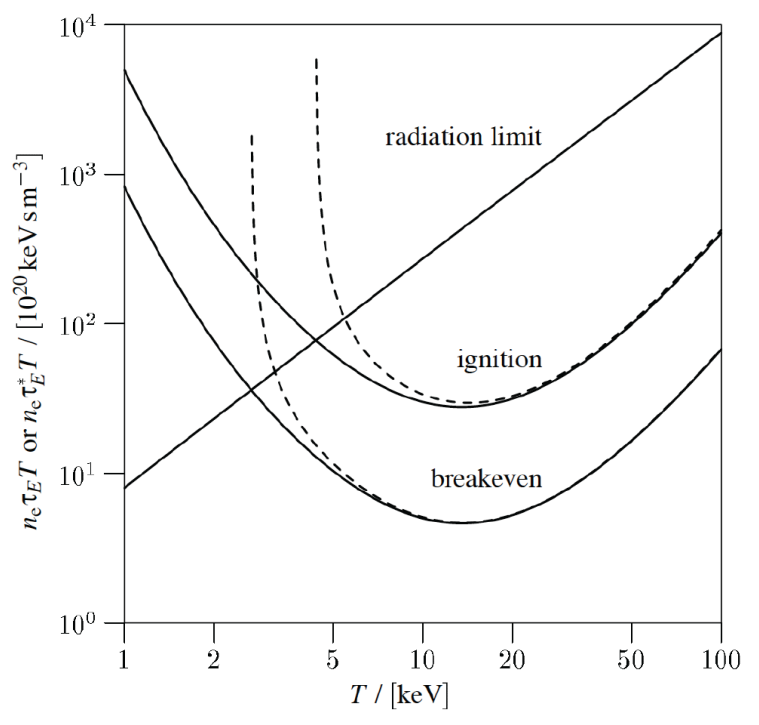

Figure 4: Curves $n_{e} \tau_{\mathrm{E}} T=f_{1}(Q ; T)$ (dashed lines) and $n_{e} \tau_{\mathrm{E}}^{*} T=f_{2}(Q ; T)$ (solid lines) for ideal ignition and ideal break-even. Also shown is the radiation limit (only relevant for the description by $\tau_{\mathrm{E}}^{*}$ ). (Figure adapted from Ref. [2].)

Inserting this in (44) yields the ideal conditions $n_{e} \tau_{\mathrm{E}}^{*} T=3 \cdot 10^{21} \mathrm{~m}^{-3} \mathrm{keV} \mathrm{s}$ for ignition, e.g. reached with $n=10^{20} \mathrm{~m}^{-3}, T=10 \mathrm{keV}$ and $\tau_{\mathrm{E}}^{*}=3 \mathrm{~s}$, and $n_{e} \tau_{\mathrm{E}}^{*} T=0.5 \cdot 10^{21} \mathrm{~m}^{-3} \mathrm{keV}$ s for break-even.

\section{B. Non-ideal ignition and break-even}

We shall now discuss the influence of the helium ash and impurities on the conditions for ignition and break-even.

For $\tau_{\alpha} \neq 0$ from (35) we also get $c_{\alpha} \neq 0$, and since according to (15) each $\alpha$-particle displaces two fuel particles and according to (19) radiates twice as much as the two together, too high alpha particle concentrations will inevitably cause the nuclear fire to suffocate. Thus, welcome as they are with respect to heating, the alpha particles may lead to a dangerous fuel dilution and provide a rather unpleasant pollution if they become too numerous. It is therefore important that they disappear due to diffusion and convection, thereby unfortunately being accompanied by fuel particles.

Diffusion and convection are the only loss mechanisms for particles, and there is no mechanism that could be compared with the loss of energy by radiation. Although the mechanisms of particle and of energy diffusion are quite different, there is a strong coupling between them. The scaling ansatz[1,2]

$$
\tau_{p} / \tau_{\mathrm{E}}=\rho=\text { const. }
$$

appears as a good approximation for the helium ash particles in the plasma core because in this particular

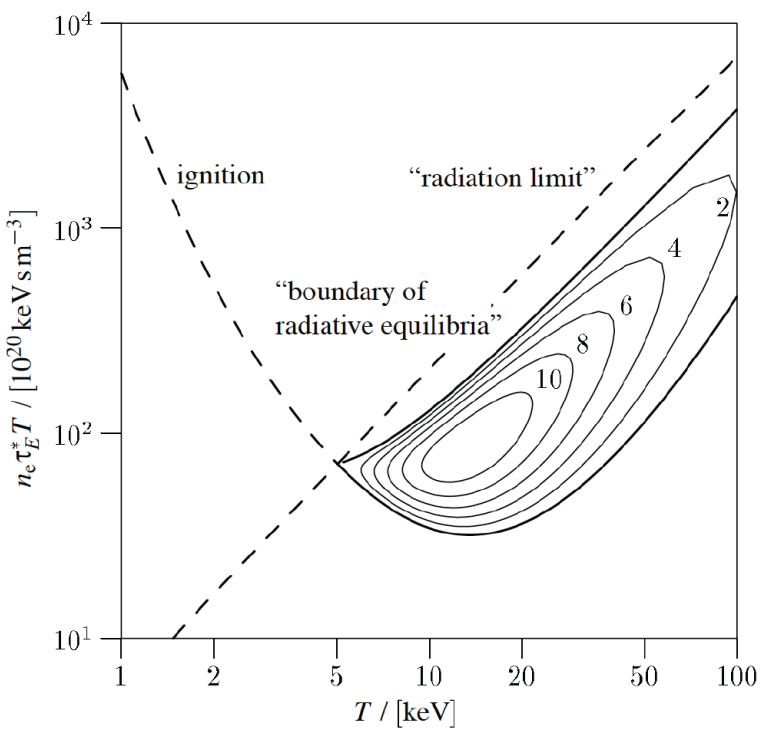

Figure 5: Curves $n_{e} \tau_{\mathrm{E}}^{*} T=f(T)$ for non-ideal ignited equilibria, radiation limit, and boundary of radiative equilibria assuming an impurity concentration of $f_{Z}=$ $2 \%$ beryllium $(Z=4)$. (Fig. adapted from Ref. [2].)

case (distinct from other species in the plasma) the particle and energy source profiles are identical. Since particles are somewhat better confined than energy, a value $\geq 5$ is expected for the ratio.

The statements made above can now be quantified by solving equations (35)-(36) together with (28) and the scaling ansatz (46). After $c_{\alpha}$ is eliminated from the equations, one can again derive an equation for $n_{e} \tau_{\mathrm{E}} T$ this time as a function of $T$ and, that can be put into the form $[2]$

$$
\rho=\rho\left(n_{e} \tau_{\mathrm{E}} T, T\right)
$$

Figure 5 shows the ignition curves $\rho=$ const numerically obtained from this for $Q=\infty$. For $\rho=0$ (corresponding to $\tau_{\alpha}=0$ ) our previous ideal curves are recovered. For $\rho>0$ one obtains closed ignition curves, and it was shown in Ref. [2] that one also obtains closed ignition curves for $n_{e} \tau_{\mathrm{E}}^{*} T(T)$ if the scaling assumption (46) with $\tau_{\mathrm{E}}$ is being kept. The most important outcome of these calculations is that ignited equilibria exist in a pure D-T plasma only for $\rho l e q 15$ (or $\rho \leq 10$ for an impurity concentration of $2 \%$ beryllium). If $\rho$ becomes larger, the helium concentration becomes too large and ignition is impossible as predicted by our qualitative arguments. However, the helium concentration requirement is relaxed if elastic scattering by collisions between helium and $\mathrm{D} / \mathrm{T}$ ions are taken into account[3]. The ignition curves shrink in size with $\rho$ even faster with increasing $Z$ and $c_{Z}$. Modelling of impurity seeded ITER discharges[7] has shown that the interplay be- 

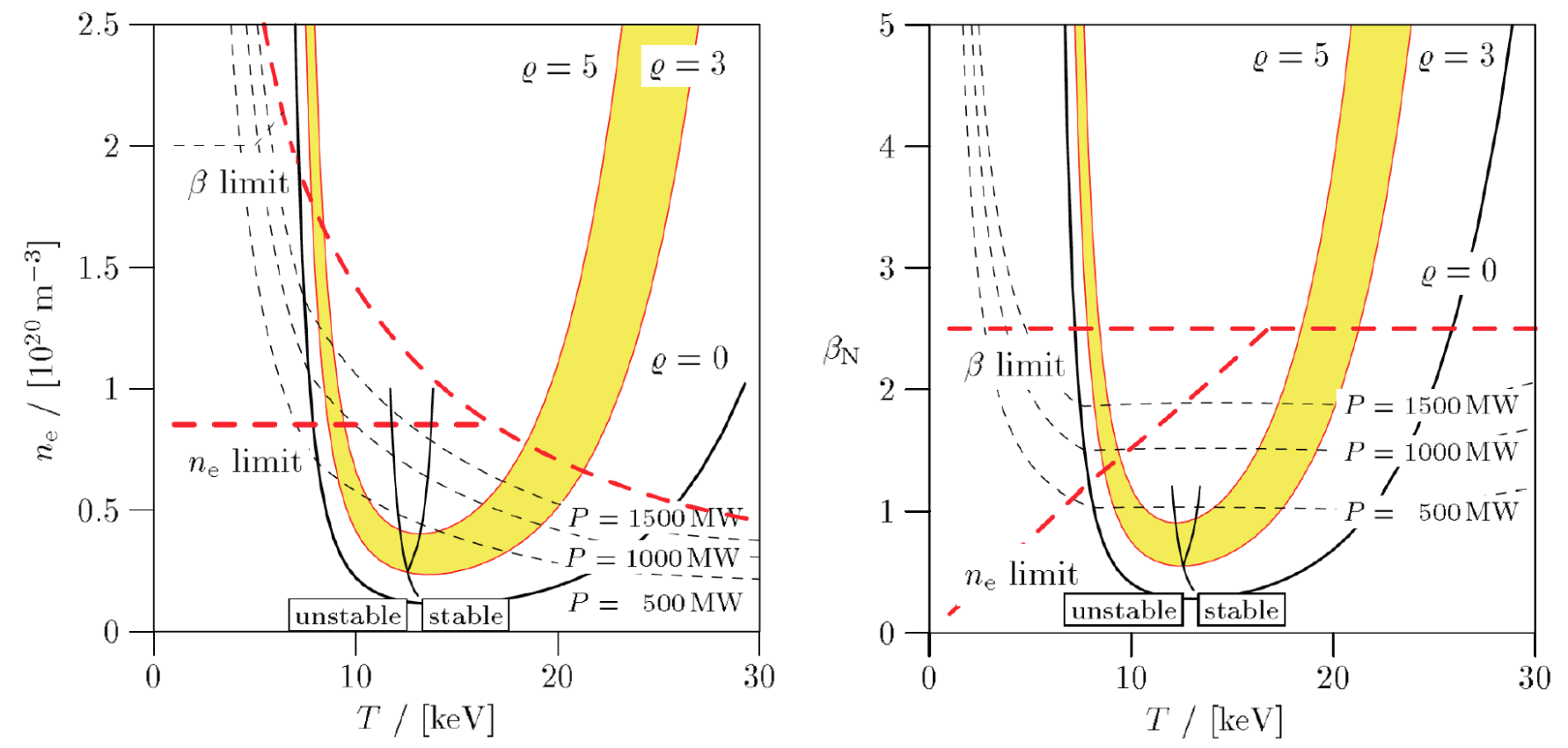

Figure 6: Ignition curve $\rho=5$ (together with curves $\rho=3$ and 0 for comparison) (a) in a $n, T$-plane and (b) in a $\beta_{N}, T$-plane. $\beta_{N}=\beta /(I / a B)$ is the so-called normalized beta). In both diagrams the boundary of stability with respect to thermal instabilities is also shown. The stable regime is to the right of all stability curves. (Figures taken from Ref. [11].).

tween He and sputtered impurities may under certain circumstances result in a rather weak dependence of $Q$ on He confinement. This would relieve the concern about helium ash removal.

\section{ITER CONFINEMENT SCALING LAWS AND TRANSFORMATION OF IGNITION CURVES TO THE N, T AND $\beta$, T PLANE}

The initially designed Ignition ITER has been replaced by a High-Q ITER, the construction of which in Cadarache has been decided in June 2005. The design of the High-Q ITER does not preclude the possibility of ignition but the objective is extended burn with $Q \geq 10$ and with a duration sufficient to reach stationary conditions with respect to the characteristic time scales. Furthermore, the design also aims at demonstrating steadystate operation using non-inductive current drive with $Q>5$.

For the planning of a burning plasma experiment like ITER it is important to have some idea about what confinement properties may be expected. Theoretically plasma transport is a very difficult and not yet satisfactorily solved problem, so the answers to this question must be essentially extrapolated from experimental data. In huge international databases the transport properties of many different tokamaks under many different circumstances have been collected and evaluated, applying as constraints certain theoretical criteria $[13,4,5]$. One expects that the energy confinement time $\tau_{\mathrm{E}}$ will depend on design parameters according to scaling laws such as ITER 89-P [6],

$$
\tau_{\mathrm{E}}=0.048 f_{H} M^{0.5} I^{0.85} B^{0.2} R^{1.2} a^{0.3} \kappa^{0.5} P^{-0.5} n_{e}^{0.1}
$$

where $f_{H}$ is the H-mode enhancement factor $\left(f_{H}=2.0\right.$ in Fig. 6), $M$ the isotopic mass (2.5 for a 50:50 D-T mixture), $I$ the plasma current in MA, $B$ the toroidal magnetic field in Teslas, $R$ and $a$ the major and minor tokamak radius in meters, $\kappa$ the elongation of the plasma cross-section, $n_{e}$ the electron density in $10^{20} \mathrm{~m}^{-3}$, and $P=P_{\mathrm{QH}}+P_{\text {ext }}+P_{\alpha}$ the net heating power in MW. Using the equilibrium equation (36), $P$ can be replaced by $\frac{3}{2} n_{\text {tot }} T / \tau_{\mathrm{E}}$ and (48) rewritten as:

$$
\tau_{\mathrm{E}}=\left(0.048 f_{H} M^{0.5} I^{0.85} B^{0.2} R^{1.2} a^{0.3} \kappa^{0.5}\right)^{2} n_{e}^{-0.8} T^{-1.0}
$$

With this relation the ignition contours (47) can be translated from the $n_{e} \tau_{\mathrm{E}} T, T$-plane directly into the $n_{e}, T$-plane (for details see Ref. [10]; note, however, that there the ITER scaling laws where applied to the energy confinement time $\tau_{\mathrm{E}}^{*}$ including radiation losses). Fig. 6(a) shows the "ignition curve" $\rho=5$ (together with $\rho=3$ and $\rho=0$ for comparison) in a $n_{e}, T$-plane, and using $\beta=n_{\text {tot }} k T B^{2} / 2 \mu_{0}$ a similar diagram can be 
obtained in the $\beta, T$-plane (see Fig. 6(b)). The advantage of representing the ignition curve as $n_{e}=n_{e}(T)$ or $\beta=\beta(T)$ is that the impact of plasma stability limits like the $\beta$-limit or the Greenwald density limit can immediately be seen.[8]

\section{BURN STABILITY}

In order to determine the stability of the burn equilibria with respect to thermal instabilities one has to solve the time dependent equations (23)-(27) for perturbations of the equilibrium states. This has extensively been done in Ref. [10], and here only the most important results are quoted. One problem arising in this context is, how to treat the confinement times during the evolution of instabilities. One possibility would be to keep them constant at their equilibrium values. Since the typical growth times of instabilities turn out to be several seconds under this assumption, it appears reasonable to assume the validity of the scaling law (49) also during this time dependent process because the plasma has time enough to adapt to these conditions which were originally derived for equilibrium states. Redoing the stability calculations with these adapted confinement times appreciably changes the stability behaviour, which for this case is shown in Figs. 6 (a) and (b). Stable behaviour is obtained to the right of the stability boundaries shown in the diagram. States to the left are unstable and undergo a transition to some state on the right branch of the corresponding ignition curve $\rho=$ const.

\section{LAWSON CRITERION AND REACTOR EFFI- CIENCY CRITERION}

If the plasma of a fusion reactor is ignited, this does not imply that there is also a net energy gain, because there are energy losses during the initial heating phase, and also energy is needed for feeding auxiliary devices to keep the reactor running. The first one to consider problems of this kind was Lawson who, in 1957, formulated the so-called Lawson criterion[9]. He asked the question: When does a fusion reactor deliver so much energy that it can run self-sustained, i.e. when does it neither need nor deliver energy? However, in this calculation Lawson neglected -particle heating, assumed that the plasma was heated from an external source, took for the discharge pulse length, and took only account of hydrogen bremsstrahlung radiation (which is small in a tokamak plasma).

In order to answer Lawsons question, we consider the sum of the internal plasma energy and the energy released in the form of radiation and fusion energy during the burn time, all expressed as specific quantities per volume,

$$
\begin{gathered}
e_{\mathrm{th}}+e_{\mathrm{rad}}+e_{\mathrm{fus}} \\
e_{\mathrm{th}}=3 n_{e} k T, e_{\mathrm{rad}}=C_{B} g_{\mathrm{ff}} n_{e}^{2} \sqrt{T} \tau_{b} \\
e_{\mathrm{fus}} \frac{1}{4} n_{e}^{2}\langle\sigma v\rangle E_{\mathrm{fus}} \tau_{b}
\end{gathered}
$$

This sum of energies is converted with efficiency $\eta_{\mathrm{th}}$, and in a self-sustained power station it supplies the thermal energy of the plasma and the radiation losses:

$$
\left(e_{\mathrm{th}}+e_{\mathrm{rad}}+e_{\mathrm{fus}}\right) \eta_{\mathrm{th}}=e_{\mathrm{th}}+e_{\mathrm{rad}}
$$

After the explicit expressions for the different energy terms are inserted, one can solve with respect to $n_{e} \tau_{b}$ to obtain the Lawson criterion

$$
n_{e} \tau_{b} \frac{12 k T}{\langle\sigma v\rangle E_{\mathrm{fus}} \eta_{\mathrm{th}} /\left(1-\eta_{\mathrm{th}}\right)-4 C_{B} g_{\mathrm{ff}} n_{e}^{2} \sqrt{T}}
$$

Similarly one can ask the question: When does a reactor yield the efficiency $\eta$ ? In order to answer this question we assume a pulsed operation of the reactor with a start-up phase of duration $\tau_{h}$ for heating the plasma to ignition, and a burning time $\tau_{b}$ with stationary conditions at temperature $T$. In the start-up phase for each volume element a heating energy $e_{h}$ must be supplied externally, from which a fraction

$$
e_{\alpha}=\eta_{a} e_{h}
$$

is absorbed by the plasma for providing its internal energy and compensating all heat losses (transport and radiation). The net efficiency of the power station is defined through:

$$
\eta=e_{\text {net }} / e_{\text {fus }}
$$

where $e_{\text {fus }}$ is the total fusion energy gain per volume (at present, probably not all fusion energy delivered to the alpha particles can be envisaged for conversion), and $e_{\text {net }}$ is the energy per volume that can be supplied to the mains as electricity. Considering all important energy flows in the reactor station, the following reactor efficiency criterion can be derived:

$$
n_{e} \tau_{b}=\frac{1}{\eta_{a}\left(\eta_{\mathrm{eff}}-\eta\right)} \frac{12 k T\left(1+\tau_{h} / \tau_{\mathrm{E}, h}^{*}\right)}{\langle\sigma v\rangle E_{\mathrm{fus}}}
$$

where $\eta_{\mathrm{eff}} \approx \eta_{\mathrm{th}} \approx 1 / 3$. We can combine this efficiency criterion with the corresponding ideal ignition criterion (44). Dividing the first by the second yields (for $Q=\infty$ )

$$
\frac{\tau_{b}}{\tau_{\mathrm{E}}^{*}}=\frac{\left(1+\tau_{h} / \tau_{\mathrm{E}, h}^{*}\right)}{5 \eta_{a} \eta_{\mathrm{eff}}\left(1-\eta / \eta_{\mathrm{eff}}\right)}
$$

where $E_{\alpha} / E_{\text {fus }}=1 / 5$ was used. This shows that the factor by which the burning time $\tau_{b}$ must exceed the 
energy confinement time $\tau_{\mathrm{E}}^{*}$ is independent of the temperature.

Assuming $\tau_{h} \approx \tau_{\mathrm{E}, \mathrm{h}}^{*}$ and $\eta_{a} / \eta_{\mathrm{eff}} \approx 1 / 20$ we get $\tau_{b} \approx 8 \tau_{\mathrm{E}}^{*} /\left(1-\eta / \eta_{\mathrm{eff}}\right)$.

For $\eta=\eta_{\text {eff }}=0.95$ this yields $\tau_{b} \approx 160 \tau_{\mathrm{E}}^{*}$ or $\tau_{b} \approx 560 \mathrm{~s}$ for $\tau_{\mathrm{E}}^{*}=3.5 \mathrm{~s}$ as expected in a fusion reactor.

In fact much longer burn times will be required for other reasons: A reactor must last for about 25 years at least in order to repay for the large expenses that are needed for its construction. A burn time of $200 \mathrm{~s}$ only would imply about $4 \times 10^{6}$ start-ups and thus changes between hot and cold during its lifetime. This is more than the reactor will stand according to all technical experience. A reasonable number of changes will be no more than about 100 000. In that case a burning cycle would have to last for about $2 \mathrm{~h}$ in order to sum up to a life time of 25 years.

\section{REFERENCES}

[1] G. H. WOLF D. REITER and H. KEVER. Burn condition, helium particle confinement and exhaust efficiency. Nucl. Fusion, (30):2141-2155, 1990.

[2] D. REITER E. REBHAN, U. VIETH and G. H. WOLF. Effectof helium concentration on ignition curves with energy confinement time including radiation losses. Nucl. Fusion, (36):264-269, 1996.

[3] H.KUBO et al. The spectral profile of the hei singlet line $(667.82 \mathrm{~nm})$ emitted from the divertor region of jt-60u. Plasma Phys. Control.Fusion, 41: 747-757, 1999.

[4] J. P. CHRISTIANSEN et al. Global energy confinement h-mode database for iter. Nucl. Fusion, (32):291-338, 1992.

[5] K. THOMSEN et al. Iter h-mode confinement database update. Nucl. Fusion, (34):131-167, 1994.

[6] P. N. YUSHMANOV et al. Scalings for tokamak energy confinement. Nucl. Fusion, (30):1999-2006, 1990.

[7] R.ZAGORSKI et al G.TELESCA. Modelling of radiative power exhaust by sputtered and seeded impurities in fusion reactors with carbon and molybdenum target plates. Nucl. Fusion, (30):16251637, 2007.

[8] H.R. KOSLOWSKI. Operational limits and limiting instabilities in tokamak machines. this volume.

[9] J. D. LAWSON. Some criteria for a power producing thermonuclear reactor. Proc. Phys. Soc., B 70: 6-10, 1957.
[10] REBHAN and U. VIETH. Burn stability and safe operating regime of a tokamak reactor with iter scaling. Nucl. Fusion, 37:251-270, 1997.

[11] E. REBHAN and U. VIETH. Parameter dependence of the operating regime and performance of d-t tokamak reactors in a current-versus-size diagram. In Proc. 24th Eur. Conf. Berchtesgaden, volume 21A, page 1029, 1997.

[12] D. REITER. Recycling and transport of neutrals. this volume.

[13] J. WESSON. Tokamaks. Oxford Engineering Science Series No. 48. Clarendon Press, third edition edition, 2004. 